\title{
Disk formation during collapse of magnetized protostellar cores ${ }^{\star}$
}

\author{
P. Hennebelle ${ }^{1}$ and A. Ciardi ${ }^{1,2}$ \\ 1 Laboratoire de radioastronomie, UMR 8112 du CNRS, École normale supérieure et Observatoire de Paris, 24 rue Lhomond, \\ 75231 Paris Cedex 05, France \\ e-mail: patrick.hennebelle@ens.fr \\ ${ }^{2}$ Laboratoire de Physique des Plasmas, UMR 7648 du CNRS, 10-12 Avenue de l'Europe, 78140 Velizy, France
}

Received 29 July 2009 / Accepted 14 September 2009

\section{ABSTRACT}

\begin{abstract}
Context. In the context of star and planet formation, understanding the formation of disks is of fundamental importance. Aims. Previous studies found that the magnetic field has a very strong impact on the collapse of a prestellar cloud, by possibly suppressing the formation of a disk even for relatively modest values of the magnetic intensity. Since observations infer that cores have a substantial level of magnetization, this raises the question of how disks form. However, most studies have been restricted to the case in which the initial angle, $\alpha$, between the magnetic field and the rotation axis equals $0^{\circ}$. Here we explore and analyse the influence of non aligned configurations on disk formation.

Methods. We perform 3D ideal MHD, AMR numerical simulations for various values of $\mu$, the ratio of the mass-to-flux to the critical mass-to-flux, and various values of $\alpha$.

Results. We find that disks form more easily as $\alpha$ increases from 0 to $90^{\circ}$. We propose that as the magnetized pseudo-disks become thicker with increasing $\alpha$, the magnetic braking efficiency is lowered. We also find that even small values of $\alpha\left(\simeq 10-20^{\circ}\right)$ show significant differences with the aligned case.

Conclusions. Within the framework of ideal MHD, and for our choice of initial conditions, centrifugally supported disks cannot form for values of $\mu$ smaller than $\simeq 3$ when the magnetic field and the rotation axis are perpendicular, and smaller than about $\simeq 5-10$ when they are perfectly aligned.
\end{abstract}

Key words. magnetohydrodynamics (MHD) - instabilities - ISM: kinematics and dynamics - ISM: clouds - ISM: structure star: formation

\section{Introduction}

Understanding the formation of protostellar disks is an important issue in the context of star and planet formation. The presence of circumstellar disks is well established around T-Tauri stars (Watson et al. 2007), as well as around younger class I and II protostars. However, because of difficulties in disentangling the emission of the disk and envelope, the existence of disks around class 0 objects remains a matter of debate (Mundy et al. 2000; Belloche et al. 2002; Jorgensen et al. 2007).

Starting with a level of rotation consistent with observations, hydrodynamical numerical simulations discovered that centrifugally supported disks form with characteristic diameters of the order of a few hundred AU (Matsumoto \& Hanawa 2003; Hennebelle et al. 2004; Goodwin et al. 2007; Commerçon et al. 2008). These structures are the unavoidable consequence of angular momentum conservation during the collapse phase. In addition, since these disks are massive and strongly selfgravitating, they are prone to gravitational instability and have often been found to fragment, leading to the formation of a small cluster of stars.

However, magnetic fields appear to have an important impact. Magnetohydrodynamic (MHD) simulations (e.g., Machida et al. 2005; Banerjee \& Pudritz 2006) find that even for modest values of the magnetic intensity, disk formation

\footnotetext{
* Appendix A is only available in electronic form at http://www . aanda.org
}

can be suppressed by magnetic braking (Shu et al. 1987; Mouschovias 1991; Basu \& Mouschovias 1995; Galli et al. 2006) which transports angular momentum from the inner parts of the cloud towards its outer regions (Allen et al. 2003; Fromang et al. 2006; Price \& Bate 2007; Hennebelle \& Fromang 2008, hereafter HF08; Mellon \& Li 2008, 2009).

The typical values of $\mu$, the ratio of the mass-to-flux to the critical mass-to-flux ratio, at which the formation of a rotationally supported disk is suppressed, vary from one study to another; probably because of different choices of initial conditions. For example, while Mellon \& Li (2008) estimate that the value of $\mu$, below which disks do not form, is larger than 10 , Price $\&$ Bate (2007) and HF08 find that this occurs when $\mu<5-10$. A broad distribution of $\mu$ has been inferred from observations, but most cores have magnetic fields corresponding to values of $\mu$ typically smaller than 5 (Crutcher 1999). This raises a fundamental question about how disks can form in spite of the relatively high degree of magnetization.

Most theoretical studies have been performed by assuming a simple initial configuration in which the magnetic field and the rotation axis are parallel. Notable exceptions are the works of Machida et al. (2006), who explored the influence of a non zero angle between the magnetic field axis and the rotation axis, and Price \& Bate (2007) who considered the case where the two axes are perpendicular. However, none of these works focused on the problem of disk formation and magnetic braking in great details, which are the topics of the present work. 
The paper is organised as follows: in Sect. 2, we analyse the magnetic braking emphasizing the importance of the initial angle, $\alpha$; in Sect. 3 numerical simulations of collapsing cores for various values of $\alpha$ and $\mu$ are presented; Sect. 4 concludes the paper.

\section{Magnetic braking: physical analysis}

The classical analysis of magnetic braking, presented in Appendix, assumes that there is a clear distinction between the cloud and the intercloud medium. However, inside a collapsing core the situation differs a priori. Density gradients (typically $\rho \propto r^{-2}$ ) throughout the cloud make it difficult to discriminate between its dense inner parts and the diffuse external medium. It is therefore not entirely clear to what extent the classical analysis may be applied to understand the magnetic braking operating inside a collapsing dense core. Indeed various studies have already demonstrated that internal magnetic braking is more complex (e.g., Galli et al. 2006; HF08).

A simple analysis of the terms responsible for angular momentum transport through the cloud is elucidating. We consider a collapsing cloud permeated by a uniform magnetic field, $B_{z}$. Rotation with respect to the $z$-axis generates a toroidal field, $B_{\theta}$ ( $r, \theta$ and $z$ are cylindrical coordinates) and we have

$\frac{v_{\theta}}{\tau_{\text {brak }}} \simeq \frac{B_{z} B_{\theta}}{4 \pi \rho h}, \frac{B_{\theta}}{\tau_{\text {brak }}} \simeq \frac{B_{z} v_{\theta}}{h}$

where $\tau_{\text {brak }}$ is the braking time and $h$ is the typical scale-height. Combining these two equations, we obtain

$\tau_{\text {brak }} \simeq \frac{\sqrt{4 \pi \rho h^{2}}}{B_{z}}$,

which clearly shows that the braking time is shorter as, $h$, decreases. Assuming that the column density remains roughly constant, we find that $\tau_{\text {brak }} \propto h^{1 / 2}$.

As discussed in Galli \& Shu (1993), Li \& Shu (1996) and HF08, a magnetized cloud flattens along the field lines producing a thin magnetic pseudo-disk, which unlike centrifugally supported disks, is dynamically collapsing. We can relate the scaleheight of the pseudo-disk, $h$, to the angle $\alpha$. For an aligned system, assuming mechanical equilibrium along the $z$-axis, one can infer the relation (see HF08 for details)

$\rho_{\mathrm{c}} c_{\mathrm{s}}^{2} \simeq B_{\mathrm{r}}^{2} / 8 \pi+\left(\partial_{z} \phi\right)^{2}$,

which reflects that the gravity and magnetic pressure compress the gas along the $z$-axis while the thermal pressure resists the compression. In this equation $B_{\mathrm{r}}$, and the gravitational force $\partial_{z} \phi$, are estimated just above the disk $(z \simeq h)$ while $\rho_{\mathrm{c}}$ is the equatorial $(z \simeq 0$ ) gas density. As shown in HF08, for values of $\mu$ lower than 5 , the magnetic pressure produces a very significant density enhancement (typically a factor 10 or more) in the equatorial plane and very stiff gradients along the $z$ direction. We now consider a cloud that rotates along an axis $o z^{\prime}$, belonging to the $x-z$ plane, such that the angle, $\alpha$, between $o z$ and $o z^{\prime}$ is small. We define $\Omega$ the rotation speed. The rotation can be decomposed into two components; one is a rotation about the $z$-axis at a velocity $\Omega \cos (\alpha)$ and the other about the $x$-axis at a (time dependent) velocity $\sim \sin (\alpha)$. As a consequence, the magnetic field lines are stretched. As in the aligned case, a toroidal component is created by the rotation with respect to $o z$ but another component, $B_{y} \simeq B_{z} \Omega \sin (\alpha) \tau_{\mathrm{ff}}$, is also generated by the rotation with respect to $o x$, where $\tau_{\mathrm{ff}}$ is the cloud freefall time. Therefore, unlike the aligned case, the components of the magnetic field parallel to the $x-y$ plane do not vanish in the equatorial plane. Assuming that the angle $\alpha$ and the global cloud rotation are small, the magnetic field remains roughly unchanged with the exception of its $y$-component. The mechanical equilibrium equation becomes

$\rho_{\mathrm{c}} c_{\mathrm{s}}^{2}+B_{y}^{2} / 8 \pi \simeq B_{\mathrm{r}}^{2} / 8 \pi+\left(\partial_{z} \phi\right)^{2}$,

where $B_{\mathrm{r}}$ is the radial field corresponding to the case without rotation. Equation (4) shows that the $B_{y}$ component generated by the cloud rotation limits the magnetic compression along the $z$-axis. Therefore, the typical width of the pseudo disk, $h$, increases as $\alpha$ increases, and the efficiency of the magnetic braking $\left(\propto h^{-1 / 2}\right)$ is consequently reduced. In addition, since the rotation axis is not parallel to the $z$-axis, the rotation itself will also tend to impede the contraction along the $z$-direction.

\section{Numerical simulations}

\subsection{Numerical setup}

To carry out our numerical simulations, we used the AMR code Ramses (Teyssier 2002; Fromang et al. 2006). Throughout the simulations, the Jeans length is resolved with at least 10 cells. An HLLD solver is employed. The initial conditions consist of a 1 solar mass spherical cloud whose profile, given by $\rho(r)=$ $\rho_{\mathrm{c}} /\left(1+\left(r / r_{0}\right)^{2}\right)$, resembles the observed cores. We impose a density contrast of 10 between the central density $\rho_{\mathrm{c}}=8 \times 10^{6} \mathrm{~cm}^{-3}$ and the edge density, $\rho_{\mathrm{e}}$. Outside the cloud, the gas has a density of $\rho_{\mathrm{e}} / 10$ and it is in pressure equilibrium with the cloud edge. As in HF08, we use a barotropic equation of state. The cloud is initially in solid body rotation and threaded by a magnetic field, along the $z$-axis, whose intensity is proportional to the total column density through the cloud. The rotation axis is in the $x-z$ plane and at an angle $\alpha$ with respect to the magnetic field. The ratio of rotation to gravitational energy is equal to $\beta \simeq 0.03$, while the degree of magnetization is determined by the parameter $\mu$. The initial thermal to gravitational energy ratio is $\sim 0.25$.

\subsection{Results}

For various values of $\mu$ and $\alpha$, Fig. 1 shows the density and velocity distribution in the $x-y$ plane (first row) and $y-z$ plane (second row). These correspond to the initial direction of the magnetic field being respectively perpendicular and parallel to the plane of the snapshot. Note that the rotation axis is also either perpendicular or parallel, except in the case of $\alpha=20^{\circ}$ for which it is slightly tilted. At the time of the snapshots, the mass of the gas denser than $10^{10} \mathrm{~cm}^{-3}$ (the density at which the gas becomes adiabatic) is $\sim 0.15 M_{\odot}$, while the mass of the gas denser than $10^{13} \mathrm{~cm}^{-3}$ is $\sim 0.1 M_{\odot}$.

The first two columns show results for the cases $\mu=16$ and $\mu=5$, respectively, with $\alpha=0^{\circ}$. In the $\mu=16$ case, a dense disk whose diameter is $\sim 200 \mathrm{AU}$ forms around the central object. The size of the disk is estimated by determining the position at which the velocity is roughly perpendicular to the disk radius, so that the rotation velocity dominates over the infall motions. The cut across the $y-z$ plane also shows an outflow being launched perpendicularly to the disk. In the $\mu=5$ case, no disk of significant size forms. The gas instead falls directly towards the central thermally supported core. The entire configuration is unstable leading to significant departures from the axisymmetric configuration. The cut across the $y$ - $z$ plane also shows the thin pseudodisk $\left(|y|>200 \mathrm{AU},|z| \simeq 100 \mathrm{AU}\right.$ for $\left.\rho=10^{8} \mathrm{~cm}^{-3}\right)$, which is compressed by the magnetic pressure exerted by the curved 


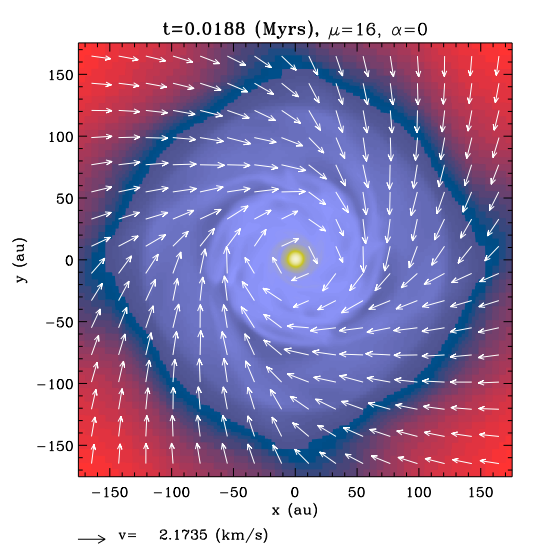

$\mathrm{t}=0.0188(\mathrm{Myrs}), \mu=16, \alpha=0$

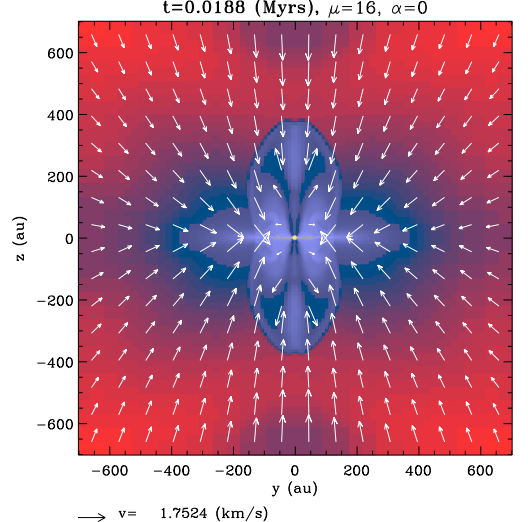

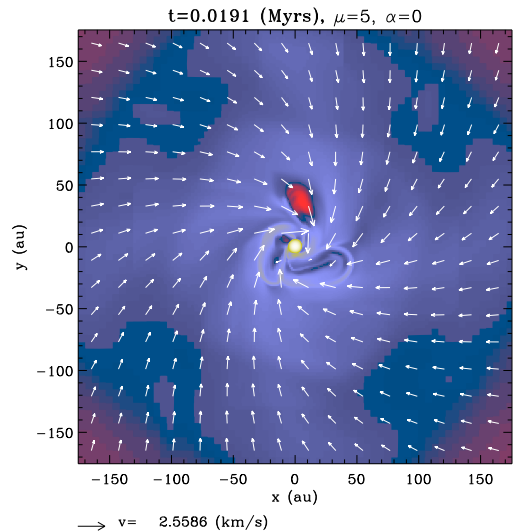

$\mathrm{t}=0.0191$ (Myrs), $\mu=5, \alpha=0$

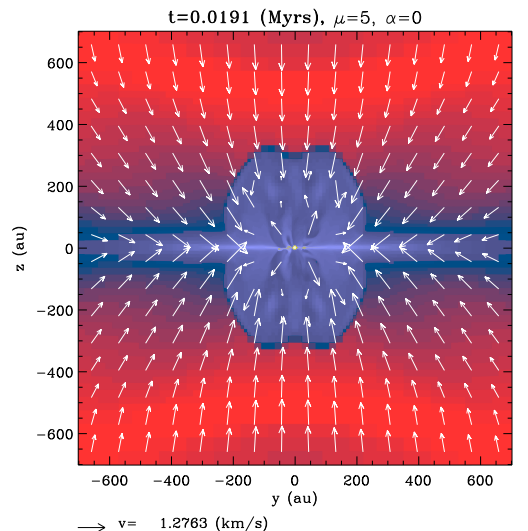

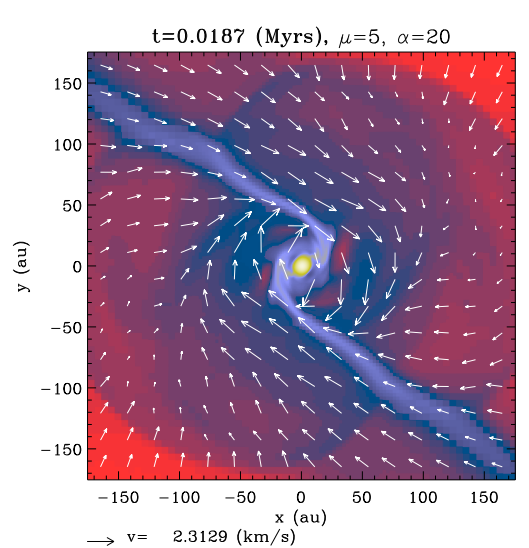
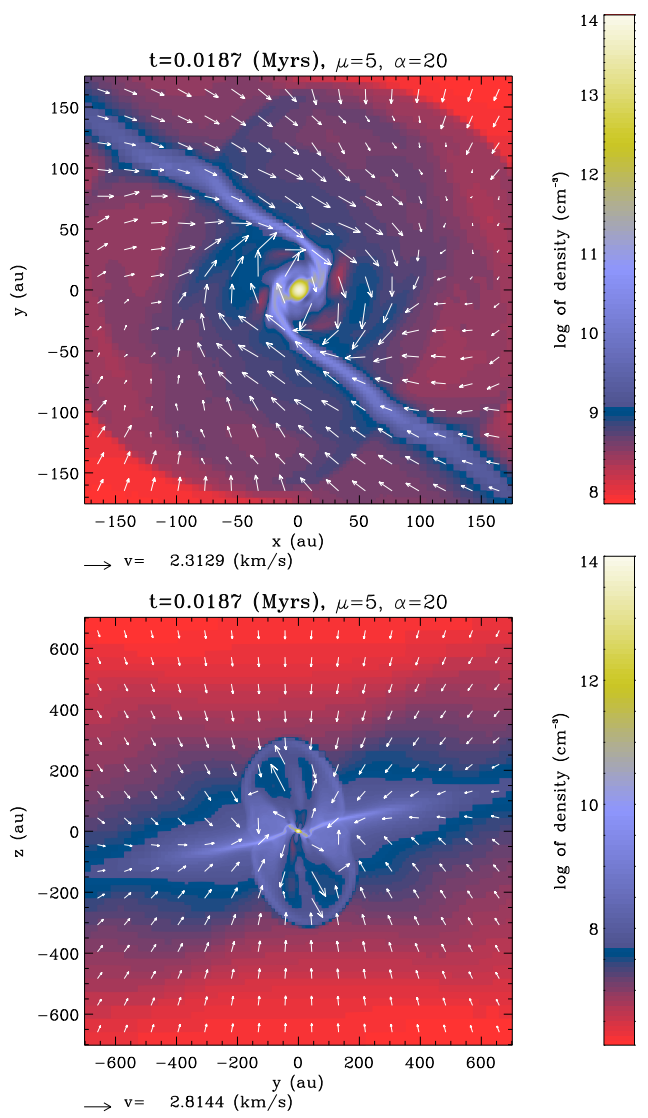

Fig. 1. Density and velocity profiles. First column is for $\mu=16$ and $\alpha=0^{\circ}$, second column is for $\mu=5$ and $\alpha=0^{\circ}$, while third column is for $\mu=5$ and $\alpha=20^{\circ}$. The first row shows the $x-y$ plane (therefore perpendicular to the magnetic field direction) and a zoomed image of the centrifugally supported disk (when it exists). The second row shows both the $y$ - $z$ plane and the more extended magnetized pseudo-disk seen edge on $(|y|>200 \mathrm{AU})$.

field lines. The top panel of the third column $\left(\mu=5, \alpha=20^{\circ}\right)$ shows that a disk-like structure of $\sim 150$ AU forms around the central object (as shown by the velocity field, rotation dominates over infall in this structure). We note that the structure appears to be non-axisymmetric and that the entire velocity field is more complex than in the first case $\left(\mu=16, \alpha=0^{\circ}\right)$. For $\mu=5$ with $\alpha=20^{\circ}$ (bottom panel of third column) the pseudo disk, visible at $|y|>200 \mathrm{AU}$, is twisted and overall thicker by a factor of $\sim 2-3$ than the case $\mu=5, \alpha=0^{\circ}$ (bottom panel of the second column). Additionally, we have performed simulations of the case $\alpha=10^{\circ}$ for which we also find a disk. According to the analysis presented in Sect. 2, we believe that the thickness of the pseudo-disk is at the origin of the formation of a rotationally dominated disk-like structure, since it reduces the magnetic braking efficiency as shown by Eq. (1). To confirm that the different behaviours are indeed related to differences in the magnetic braking efficiency, we plot in Fig. 2 the quantity $\left|B_{z} B_{\theta}\right| / \rho$ which appears in Eq. (2). The cases $\theta=0^{\circ}$ (solid line), $\theta=10^{\circ}$ (dotted line) and $\theta=20^{\circ}$ (dashed line) are shown. As can be seen, the quantity $\left|B_{z} B_{\theta}\right| / \rho$ is higher, and the two peaks are closer (equivalently the gradients between the peaks are stiffer), for the aligned case; implying that the quantity $B_{z} B_{\theta} /(\rho h)$ is higher by at least $\sim 50-100 \%$ than for the two misaligned configurations $\left(B_{\theta}=0\right.$ at $z=0$, for symmetry reasons). This sudden change of behaviour as $\alpha$ increases, indicates, as suggested by Eqs. (3-4), that the aligned configuration is probably too restrictive for studying disk formation in collapsing magnetized cores.

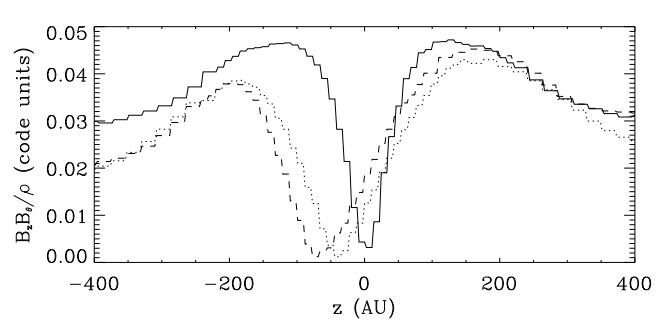

Fig. 2. $\left|B_{z} B_{\theta} / \rho\right|$ (see Eq. (1)) as a function of $z$ at $y=300 \mathrm{AU}$. Solid line is $\theta=0^{\circ}$, dotted line $\theta=10^{\circ}$ and dashed line $\theta=20^{\circ}$.

Figure 3 shows the density and velocity distributions in the $y-z$ plane for larger values of $\alpha$. The first panel illustrates the $\mu=5$ and $\alpha=60^{\circ}$ case, while the second panel shows results for $\alpha=90^{\circ}$. In both cases a disk of $\sim 150-200$ AU forms. We note that the densities in these disks are significantly lower than those observed in the $\mu=16$ and $\alpha=0^{\circ}$ case (first row of Fig. 1); their structure is clearly more complex, and we note the presence of extended spiral arms, which are almost one order of magnitude denser than in the rest of the disk. Finally, the third panel of Fig. 3 shows results for a highly magnetized cloud rotating perpendicularly to the magnetic field $\left(\mu=2, \alpha=90^{\circ}\right)$. In this case, even though the magnetic braking is less efficient than in the aligned configuration, the magnetic field is so strong that 

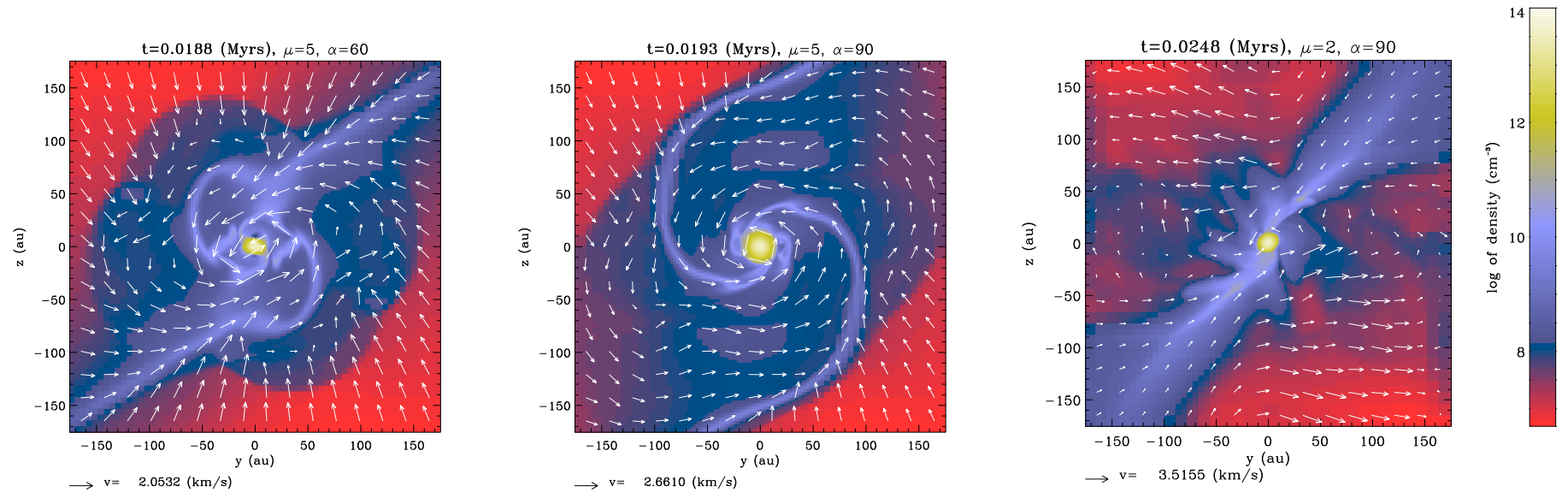

Fig. 3. Density and velocity cut through the $y-z$ plane. The centrifugally supported disk, when it exists, is seen nearly face on. First panel is for $\mu=5$ and $\alpha=60^{\circ}$, second panel is for $\mu=5$ and $\alpha=90^{\circ}$ while third panel is for $\mu=2$ and $\alpha=90^{\circ}$.

no disk forms. Interestingly, we also see that a relatively strong outflow is launched. For completeness, we also considered the case $\left(\mu=3, \alpha=90^{\circ}\right)$ for which we find that a small disk-like object of size 50-100 AU forms. Thus, we conclude that in the perpendicular case and within the framework of ideal MHD, disks can form for values of $\mu$ higher than $\simeq 3$. It seems probable to us that, given the complex field geometry induced in these non aligned configurations, ambipolar diffusion as well as ohmic dissipation may play a role in the formation of small disks.

\section{Conclusion}

To study the formation of circumstellar disks, we have performed numerical simulations of the gravitational collapse of magnetized dense prestellar cores for a range of values of $\mu$ and $\alpha$ (the initial angle between magnetic field and the rotation axis). Our main conclusions are the following. In the aligned case $\left(\alpha=0^{\circ}\right)$ disk formation is prevented for values of $\mu$ as high as $\sim 5-10$. In the non aligned cases, and even for relatively small values of $\alpha \simeq 10-20^{\circ}$, disks can form for smaller values of $\mu \simeq 4-5$, corresponding to larger magnetic field intensities. The main reason for this change of behaviour is a decrease, for increasing $\alpha$, of the magnetic braking efficiency, which is linked to an increase in the thickness of the magnetized pseudo-disk. This is caused by the magnetic field lines being twisted by the rotation about an axis parallel to the plane of the pseudo-disk. When $\alpha=90^{\circ}$, we find that disks may form for smaller values of $\mu$, as long as $\mu>2-3$, and for even lower values of $\mu$, disk formation does not seem to be possible. Nevertheless, for these highly magnetized configurations, the question of whether a disk may form at later times, or because of non-ideal MHD effects (Hosking \& Whitworth 2004; Machida et al. 2008; Mellon \& Li 2009), remains unanswered. We recall that although Belloche et al. (2002) observe a significant amount of rotation in the envelope of IRAM04191, they exclude a disk of size larger than $20 \mathrm{AU}$. This result is very difficult to explain without a magnetic field, but agrees at least qualitatively with our conclusion if the source is sufficiently magnetized (Troland \& Crutcher 2008).

Another important point is that while in the purely hydrodynamical case, and for the cloud parameters used, fragmentation occurs; we do not identify fragmentation in any of the magnetized cases explored. It is however possible that with a better treatment of the thermal processes, the magnetized disks which form in the low magnetized configurations, may also fragment.

Acknowledgements. This work was performed using HPC resources from GENCI-cines (2009-042036) and cemag. A.C. is supported by the ANR grant MAGNET and by the Marie Curie Reintegration Grant MAGPLUS. We thank Shantanu Basu as well as an anonymous referee for comments which have improved the paper significantly.

\section{References}

Allen, A., Li, Z.-Y., \& Shu, F. 2003, ApJ, 599, 363

Banerjee, R., \& Pudritz, R. 2006, ApJ, 641, 949

Basu, S., \& Mouschovias, T. 1995, ApJ, 452, 386

Belloche, A., André, P., Despois, D., et al. 2002, A\&A, 393, 927

Commerçon, B., Hennebelle, P., Audit, E., Chabrier, G., \& Teyssier, R. 2008, A\&A, 482, 371

Crutcher, R. 1999, ApJ, 520, 706

Fromang, S., Hennebelle, P., \& Teyssier, R. 2006, A\&A, 457, 371

Galli, D., \& Shu, F. 1993, ApJ, 417, 243

Galli, D., Lizano, S., Shu, F., et al. 2006, ApJ, 647, 374

Goodwin, S., Whitworth, A., \& Ward-Thompson, D. 2004, A\&A, 423, 169

Hennebelle, P., Whitworth, A., Cha, S.-H., et al. 2004, MNRAS, 340, 870

Hennebelle, P., \& Fromang, S. 2008, A\&A, 477, 9 (HF08)

Hennebelle, P., \& Teyssier, R. 2008, A\&A, 477, 25

Hosking, G., \& Whitworth, A. 2004, MNRAS, 347, 994

Jorgensen, J., Bourke, T., Myers, P., et al. 2007, ApJ, 659, 479

Li, Z-Y., \& Shu, F. 1996, ApJ, 472, 211

Machida, M., Matsumoto, T., Tomisaka, K., et al. 2005, MNRAS, 362, 369

Machida, M., Matsumoto, T., Hanawa, T., et al. 2006, ApJ, 645, 1227

Machida, M., Tomisaka, K., Matsumoto, T., et al. 2008, ApJ, 677, 327

Matsumoto, T., \& Hanawa, T. 2003, ApJ, 595, 913

Mellon, R., \& Li, Z.-Y. 2008, ApJ, 681, 1356

Mellon, R., \& Li, Z.-Y. 2009, ApJ, 698, 922

Mouschovias, T. 1991, ApJ, 373, 169

Mundy L., Looney, L., \& Welch, W. 2000, in Protostars and Planets IV (Book Tucson: University of Arisona Press, eds. Manning, V., Boss, A. P. \& Russell, S. S., 355

Price, D., \& Bate, M. 2007, MNRAS, 377, 77

Shu, F., Adams, F., \& Lizano, S., 1987, ARA\&A, 25, 23

Teyssier, R. 2002, A\&A, 385, 337

Troland, T., \& Crutcher, R. 2008, ApJ, 680, 457

Watson, A., Stapelfeldt, K., Wood, K., et al. 2007, prpl, 523 


\section{Appendix A: Classical analysis of the magnetic braking: cloud embedded into an external medium}

In the context of interstellar clouds, the classical analysis of magnetic braking (e.g.; Mouschovias 1991; Shu et al. 1987), considers a rigid and dense axisymmetric cloud. We define $\rho_{\mathrm{c}}$ to be its density, $R$ its radius and $Z$ its height. The cloud is surrounded by a diffuse inter cloud medium of density $\rho_{\text {ic }}$. The typical timescale for magnetic braking $\tau_{\mathrm{br}}$, corresponds to the time necessary for torsional Alfvén waves induced by the twisting of the magnetic field line, to propagate over a distance $l$, such that the mass of gas swept by the waves is comparable to the mass of the cloud itself. At this point, a significant fraction of the cloud angular momentum has been transferred to the intercloud medium.

Two cases can be considered. First, when the magnetic field and the rotation axis are aligned, the waves propagate along the magnetic field at the Alfvén speed $V_{\mathrm{a}}$, and $l \rho_{\mathrm{ic}} \simeq Z \rho_{\mathrm{c}}$, leading to

$\tau_{\mathrm{br}} \simeq \frac{Z}{V_{a}} \frac{\rho_{c}}{\rho_{i c}}$

The second case corresponds to the magnetic field and the rotation axis being perpendicular to each other, and the waves propagating in the equatorial plane of the cloud. The intercloud medium, which at time $t=\tau_{\mathrm{br}}$ is reached by the torsional Alfvén waves, is located in a cylinder of radius $l$ and height $Z$. In this case, $\left(\left(\tau_{\mathrm{br}} V_{\mathrm{a}}\right)^{2}-R^{2}\right) \rho_{\mathrm{ic}} \simeq R^{2} \rho_{\mathrm{c}}$, which gives

$\tau_{\mathrm{br}} \simeq \frac{R}{V_{\mathrm{a}}} \sqrt{\frac{\rho_{\mathrm{c}}}{\rho_{\mathrm{ic}}}+1}$.

Since in typical astrophysical circumstances the intercloud medium has a density that is low with respect to the cloud density, Eqs. (A.1) and (A.2) show that the braking is usually more efficient when the magnetic field is perpendicular to the rotation axis than when it is parallel. However, this conclusion is obviously correct only when $R \simeq Z$, i.e., if the cloud aspect ratio is not too different from 1 . In particular, from Eqs. (A.1)-(A.2) we see that if $Z / R \ll 1 / \sqrt{\rho_{\mathrm{c}} / \rho_{\text {ic }}}$, the braking time is shorter in the aligned case than for the perpendicular configuration.

Previous studies have demonstrated that magnetized clouds are usually very flat because of the magnetic compression exerted by the radial component of the magnetic field (see e.g.; Li \& Shu 1996, HF08). These magnetized sheets, which are called pseudo-disks, are perpendicular to the average magnetic field. In the same way, centrifugally supported disks are also very flat objects that are perpendicular to the rotation axis. For these extreme configurations, the magnetic braking time can obviously be longer when the magnetic field and the rotation axis are perpendicular than when they are parallel (depending on the respective values of $R, Z$ and $\rho_{\mathrm{c}} / \rho_{\text {ic }}$ ). Note that strictly speaking, if the magnetic field and the rotation axis are not aligned with each other, the resulting structure is fully tridimensional rather than axisymmetric.

It is interesting to compare Eqs. (A.1)-(A.2) with Eq. (2). Even though the latter is identical to the first for $\rho_{\mathrm{c}}=\rho_{\text {ic }}$, there are two major differences. First, even when $R \simeq Z$, the magnetic braking is not significantly more efficient in the perpendicular configuration than in the aligned one. The relative efficiency of the magnetic braking in the two configurations is, instead, directly proportional to the cloud aspect ratio. Second, as the cloud is compressed along the field lines, the quantity $Z / \sqrt{\rho_{\mathrm{c}}}$ is simply proportional to $Z^{1 / 2}$, implying that the magnetic braking time in the aligned configuration decreases. Again this is unlike the case of a rigid cloud embedded in a diffuse intercloud medium. 